\title{
GW23-e0509 INHIBITION OF THE ACTIVITY OF POLY (ADP-RIBOSE) POLYMERASE REDUCES HEART ISCHAEMIA/ REPERFUSION INJURY VIA SUPPRESSING JNK MEDIATED AIF TRANSLOCATION
}

doi:10.1136/heartjnl-2012-302920a.214

${ }^{1}$ Song Zhao-feng, ${ }^{2}$ Chen Dong-yu, ${ }^{1} \mathrm{Du}$ Bo, ${ }^{3} \mathrm{Ji}$ Xiao-ping, ${ }^{1}$ Song Zhao-feng. ${ }^{1}$ Department of Cardiology, Taian Central Hospital, 271000 Taian, Shandong Province, China; ${ }^{2}$ Department of Rheumatology, Taian Central Hospital, 271000 Taian, Shandong Province, China; ${ }^{3}$ Department of Cardiology, Shandong University Qilu Hospital, 250012 Jinan, Shandong Province, China

Objectives Poly (ADP-ribose) polymerase (PARP) played an important role in the pathogenesis of heart ischaemia/reperfusion (I/R) injury. The mechanisms are still not thoroughly understood. Here we investigated the effect of PARP inhibition on heart I/R injury and elucidated the underlying mechanisms.

Methods Studies were performed with I/R rats' hearts. 3,4dihydro-5-[4-(1-piperidinyl) butoxy]-1(2H)- isoquinolinone (DPQ) was used to inhibit the activate of PARP. The myocardial infarction size, cardiac function and cells apoptosis were detected. The activation of PARP, c-Jun $\mathrm{NH}_{2}$-terminal kinase (JNK) and Apoptosis-inducing factor (AIF) were evaluated during the $I / R$ protocol.

Results Heart I/R caused a significant increase in PARP, JNK and AIF activity. Administration of DPQ decreased myocardial infarction size from $60.97 \pm 4.22 \%$ to $39.03 \pm 3.94 \% \mathrm{p}<0.05$ ) and cells apoptosis from $35 \pm 5.3 \%$ to $20 \pm 4.1 \% \mathrm{p}<0.05$ ) and simultaneously improved the cardiac function. Administration of $\mathrm{DPQ}$ reduced the activation of JNK and attenuated mitochondrial-nuclear translocation of AIF. Administration of SP600125 also attenuated mitochondrial-nuclear translocation of AIF.

Conclusions Our results suggested that the inhibition of PARP was able to reduce heart I/R injury. JNK may be downstream of PARP activation and be required for PARP mediated AIF translocation. Inhibition of the activity of PARP may reduce heart $I / R$ injury via suppressing AIF translocation mediated by JNK. 